NTUA-96/00

hep-ph/0010141

\title{
Supersymmetry Breaking by Dimensional Reduction over Coset Spaces
}

\author{
P. Manousselis ${ }^{a}$ and G. Zoupanos ${ }^{b}$ \\ Physics Department, National Technical University, \\ Zografou Campus, 157 80, Athens, Greece.
}

\begin{abstract}
We study the dimensional reduction of a ten-dimensional supersymmetric $E_{8}$ gauge theory over six-dimensional coset spaces. We find that the coset space dimensional reduction over a symmetric coset space leaves the four dimensional gauge theory without any track of the original supersymmetry. On the contrary the dimensional reduction over a non symmetric coset space leads to a softly broken supersymmetric gauge theory in four dimensions. The $\mathrm{SO}_{7} / \mathrm{SO}_{6}$ and $\mathrm{G}_{2} / \mathrm{SU}(3)$ are used as representative prototypes of symmetric and non symmetric coset spaces respectively.
\end{abstract}

${ }^{a}$ e-mail address: pman@central.ntua.gr. Supported by ГГЕТ grand 97E $\Lambda / 71$.

${ }^{b}$ e-mail address: George.Zoupanos@cern.ch. Partially supported by the EU project ERBFM-RXCT960090. 


\section{Introduction}

Supersymmetry is an essential ingredient of most unification attempts in the recent years. A celebrated aspect of supersymmetry has been its role in providing a partial solution to the hierarchy problem of GUTs [1], resulting from the non renormalization theorem [2]. Since the observed low energy world is lacking of possessing supersymmetry in its known particle spectrum, supersymmetry has to be broken. In fact, the understanding of supersymmetry breaking is a fundamental open question in all supersymmetric unification schemes and in particular in superstring theories despite some attractive proposals [3]. For phenomenological purposes the supersymmetry breaking is parametrized by the corresponding soft terms which are defined as those that do not spoil the ultraviolet behaviour of supersymmetric theories 《4. In this spirit the Minimal Supersymmetric Standard Model (MSSM) has been provided with a soft supersymmetry breaking (SSB) sector parametrizing our ignorance of a convincing mechanism.

Recently a lot of interest has been triggered by the possibility that superstrings can be defined at a $\mathrm{TeV}$ scale [5]. The string tension became an arbitrary parameter and can be anywhere below the Planck scale and as low as TeV. The main advantage of having the string tension at the $\mathrm{TeV}$ scale, besides the obvious experimental interest, is that it offers an automatic protection to the gauge hierarchy [5], alternative to low energy supersymmetry [1], or dynamical electroweak symmetry breaking [6], [7], [8]. However the only vacua of string theory free of all pathologies are supersymmetric. Then the original supersymmetry of the theory, not being necessary in four dimensions, could be broken by the dimensional reduction procedure.

The weakly coupled ten-dimensional $E_{8} \times E_{8}$ supersymmetric gauge theory is one of the few to posses the advantage of anomaly freedom [9] and has been extensively used in efforts to describe quantum gravity along with the observed low energy interactions in the heterotic string framework [10]. In addition its strong coupling limit provides an interesting example of the realization of the brane picture, i.e. $E_{8}$ gauge fields and matter live on the two 10-dimensional boundaries, while gravitons propagate in the eleven-dimensional bulk [11].

Having a gauge theory defined in higher dimensions the obvious way to dimensionally reduce it is to demand that the field dependence on the extra coordinates is such that the Lagrangian is independent of them. A crude way to achieve that is to disccard the field dependence on the extra coordinates, while an elegant one is to allow for a non trivial dependence on them, but impose the condition that a symmetry transformation by an element of the isometry group $S$ of $B$ corresponds to a gauge transformation. Then the Lagrangian will be independent of the extra coordinates just because it is gauge invariant. This is the basis of the Coset Space Dimensional Reduction (CSDR) scheme [12], [13], [14], which assumes that $B$ is a compact coset space, $S / R$. The requirement that transformations of the fields under the action of the symmetry group of $S / R$ are compensated by gauge transformations lead to certain constraints on the fields. The solution of these constraints provides us with the four-dimensional unconstrained fields as well as with the gauge invariance that remains in the theory after dimensional reduction. It is interesting to note that the fields obtained using the CSDR approach are the first terms in the expansion of the D-dimensional fields 
in harmonics of the internal space $B$ and are massless after the first stage of the symmetry breaking.

The effective field theories resulting from compactification of higher dimensional theories contain also towers of massive higher harmonics (Kaluza-Klein) excitations, whose contributions at the quantum level alter the behaviour of the running couplings from logarithmic to power [15]. As a result the traditional picture of unification of couplings may change drastically [16].

Higher dimensional theories have also been studied at the quantum level using the continuous Wilson renormalization group [17] which can be formulated in any number of space-time dimensions with results in agreement with the treatment involving massive Kaluza-Klein excitations. In turn the CSDR approach can in principle be exploited in the study of higher dimensional unified field theories independently of reference to more general frameworks like string theories.

In the present work we study the dimensional reduction of a ten-dimensional supersymmetric $E_{8}$ gauge theory over a symmetric and a non symmetric six-dimensional coset space. We show that in the first case supersymmetry is broken completely by the reduction procedure, while in the second a supesymmetric gauge theory is obtained in four dimensions with a complete soft supersymmetry breaking sector.

\section{The Coset Space Dimensional Reduction}

In the Coset Space Dimensional Reduction (CSDR) scheme [12, 13], 14] one starts with a Yang-Mills-Dirac Lagrangian with gauge group $G$ defined on a $D$-dimensional spacetime $M^{D}$, with metric $g^{M N}$, which is compactified to $M^{4} \times S / R$ with $S / R$ a reductive but in general non symmetric coset space. The metric is assumed to have the form

$$
g^{M N}=\left[\begin{array}{cc}
\eta^{\mu \nu} & 0 \\
0 & -g^{a b}
\end{array}\right],
$$

where $\eta^{\mu \nu}=\operatorname{diag}(1,-1,-1,-1)$ and $g^{a b}$ is the coset space metric. We can divide the generators of $S, Q_{A}$ in two sets : the generators of $R, Q_{i}(i=1, \ldots, \operatorname{dim} R)$, and the generators of $S / R, Q_{a}(a=\operatorname{dim} R+1 \ldots, \operatorname{dim} S)$, and $\operatorname{dim} S / R=\operatorname{dim} S-\operatorname{dim} R=d$. Then the commutation relations for the generators of $S$ are the following:

$$
\begin{aligned}
{\left[Q_{i}, Q_{j}\right] } & =f_{i j}^{k} Q_{k}, \\
{\left[Q_{i}, Q_{a}\right] } & =f_{i a}^{b} Q_{b}, \\
{\left[Q_{a}, Q_{b}\right] } & =f_{a b}^{i} Q_{i}+f_{a b}^{c} Q_{c} .
\end{aligned}
$$

When $S / R$ is symmetric, $f_{a b}^{c}=0$. Let us call the coordinates of $M^{4} \times S / R$ space $z^{M}=$ $\left(x^{m}, y^{\alpha}\right)$, where $\alpha$ is a curved index of the coset, $a$ is a tangent space index and $y$ defines an element of $S$ which is a coset representative, $L(y)$. The vielbeins and connection forms are defined through the Maurer-Cartan form which takes values in the Lie algebra of $S$ :

$$
L^{-1}(y) d L(y)=e_{\alpha}^{A} Q_{A} d y^{\alpha} .
$$


Doing a computation at the origin $\mathrm{y}=0$ and using the fact that near the origin $L(y)=$ $\exp \left(y^{a} Q_{a}\right)$, we find that $e_{\alpha}^{a}=\delta_{\alpha}^{a}$ and $e_{\alpha}^{i}=0$. Now the group $S$ acts as a symmetry group on the the extra coordinates (there are $\operatorname{dim} S$ Killing vectors which generate the isometries of $S / R)$. The CSDR scheme demands that an $S$-transformation of the extra $d$ coordinates is a gauge transformation of the fields that are defined on $M^{4} \times S / R$, thus a gauge invariant Lagrangian written on this space is independent of the extra coordinates. Let us see this in more detail.

Consider a $D$-dimensional Yang-Mills-Dirac theory with gauge group $G$ defined on a manifold $M^{D}$ which as stated will be compactified to $M^{4} \times S / R, D=4+d, d=\operatorname{dim} S-\operatorname{dim} R$ :

$$
A=\int d^{4} x d^{d} y \sqrt{-g}\left[-\frac{1}{4} \operatorname{Tr}\left(F_{M N} F_{K \Lambda}\right) g^{M K} g^{N \Lambda}+\frac{i}{2} \bar{\psi} \Gamma^{M} D_{M} \psi\right]
$$

where

$$
D_{M}=\partial_{M}-\theta_{M}-A_{M}
$$

with

$$
\theta_{M}=\frac{1}{2} \theta_{M N \Lambda} \Sigma^{N \Lambda}
$$

the spin connection of $M^{D}$, and

$$
F_{M N}=\partial_{M} A_{N}-\partial_{N} A_{M}-\left[A_{M}, A_{N}\right]
$$

where $M, N$ run over the $D$-dimensional space. The fields $A_{M}$ and $\psi$ are, as explained, symmetric in the sense that any transformation under symmetries of $S / R$ is compensated by gauge transformations. The fermion fields can be in any representation $F$ of $G$ unless a further symmetry such as supersymmetry is required. So if $g(s), f(s)$ are gauge transformations in the adjoint and $F$ representations of $G$ corresponding to the transformation $s \in S$ acting on $S / R$, we require

$$
\begin{aligned}
A_{\mu}(x, y) & =g(s) A_{\mu}\left(x, s^{-1} y\right) g^{-1}(s) \\
A_{\alpha}(x, y) & =g(s) J_{\alpha}^{\beta} A_{\beta}\left(x, s^{-1} y\right) g^{-1}(s)+g(s) \partial_{\alpha} g^{-1}(s), \\
\psi(x, y) & =f(s) \Omega \psi\left(x, s^{-1} y\right) f^{-1}(s),
\end{aligned}
$$

where $J_{\beta}^{\alpha}$ is the Jacobian matrix for the transformation s and $\Omega$ is the Jacobian matrix plus a local Lorentz rotation in the tangent space which is needed for the fermions when they transform in a curved space. These conditions imply certain constraints that the $D$ dimensional fields have to obey. The solution of these constraints will provide us with the four-dimensional unconstrained fields as well as with the gauge invariance that remains in the theory after dimensional reduction. From eq.(7) it follows that the components $A_{\mu}(x, y)$ of the initial gauge field $A_{M}(x, y)$ become, after dimensional reduction, the four-dimensional gauge fields and furthermore they are independent of $y$. In addition one can find that they have to commute with the elements of the $R_{G}$ subgroup of $G$. Thus the four-dimensional gauge group $H$ is the centralizer of $R$ in $G, H=C_{G}\left(R_{G}\right)$.

Similarly, from eq.(8) the $A_{\alpha}(x, y)$ components of $A_{M}(x, y)$ denoted by $\phi_{\alpha}(x, y)$ from now on, become scalars at four dimensions. These fields transform under $R$ as a vector $v$, i.e.

$$
\begin{aligned}
S & \supset R \\
\operatorname{adj} S & =\operatorname{adj} R+v .
\end{aligned}
$$


Moreover $\phi_{\alpha}(x, y)$ act as an intertwining operator connecting induced representations of $R$ acting on $G$ and $S / R$. This implies, exploiting Schur's lemma, that the transformation properties of the fields $\phi_{\alpha}(x, y)$ under $H$ can be found if we express the adjoint representation of $G$ in terms of $R_{G} \times H$ :

$$
\begin{aligned}
G & \supset R_{G} \times H \\
\operatorname{adj} G & =(\operatorname{adj} R, 1)+(1, \operatorname{adj} H)+\sum\left(r_{i}, h_{i}\right) .
\end{aligned}
$$

Then if $v=\sum s_{i}$, where each $s_{i}$ is an irreducible representation of $R$, there survives an $h_{i}$ multiplet for every pair $\left(r_{i}, s_{i}\right)$, where $r_{i}$ and $s_{i}$ are identical irreducible representations of $R$.

Turning next to the fermion fields [18], [19], [13] we see from eq.(9) that they act as intertwining operators between induced representations acting on $G$ and the tangent space of $S / R, S O(d)$. Proceeding along similar lines as in the case of scalars to obtain the representation of $H$ under which the four dimensional fermions transform, we have to decompose the representation $F$ of the initial gauge group in which the fermions are assigned under $R_{G} \times H$, i.e.

$$
F=\sum\left(t_{i}, h_{i}\right)
$$

and the spinor of $S O(d)$ under $R$

$$
\sigma_{d}=\sum \sigma_{j}
$$

Then for each pair $t_{i}$ and $\sigma_{i}$, where $t_{i}$ and $\sigma_{i}$ are identical irreducible representations there is an $h_{i}$ multiplet of spinor fields in the four dimensional theory. In order however to obtain chiral fermions in the effective theory we have to impose further requirements. We first impose the Weyl condition in $D$ dimensions. In $D=4 n+2$ dimensions which is the case at hand, the decomposition of the left handed, say spinor under $S U(2) \times S U(2) \times S O(d)$ is

$$
\sigma_{D}=\left(2,1, \sigma_{d}\right)+\left(1,2, \bar{\sigma}_{d}\right)
$$

So we have in this case the decompositions

$$
\sigma_{d}=\sum \sigma_{k}, \bar{\sigma}_{d}=\sum \bar{\sigma}_{k}
$$

Let us start from a vector like representation $F$ for the fermions. In this case each term $\left(t_{i}, h_{i}\right)$ in $(12)$ will be either self-conjugate or it will have a partner $\left(\bar{t}_{i}, \bar{h}_{i}\right)$. According to the rule described in eqs.(12), (13) and considering $\sigma_{d}$ we will have in four dimensions lefthanded fermions transforming as $f_{L}=\sum h_{k}^{L}$. It is important to notice that since $\sigma_{d}$ is non self-conjugate $f_{L}$ is non self-conjugate too. Similarly from $\bar{\sigma}_{d}$ we will obtain the right handed representation $f_{R}=\sum \bar{h}_{k}^{R}$ but as we have assumed that $F$ is vectorlike, $\bar{h}_{k}^{R} \sim h_{k}^{L}$. Therefore there will appear two sets of Weyl fermions with the same quantum numbers under $H$. This is already a chiral theory, but still one can go further and try to impose the Majorana condition in order to eliminate the doubling of the fermionic spectrum. We should remark now that if we had started with $F$ complex, we should have again a chiral theory since in this case $\bar{h}_{k}^{R}$ is different from $h_{k}^{L}$ ( $\sigma_{d}$ non self-conjugate). Nevertheless starting with $F$ vectorlike is much more appealing and will be used in the following along with the Majorana 
condition. The Majorana condition can be imposed in $D=2,3,4+8 n$ dimensions and is given by $\psi=C(\bar{\psi})^{T}$, where $C$ is the $D$ dimensional charge conjugation matrix. Majorana and Weyl conditions are compatible in $D=4 n+2$ dimensions. Then in our case if we start with Weyl-Majorana spinors in $D=4 n+2$ dimensions we force $f_{R}$ to be the charge conjugate to $f_{L}$, thus arriving in a theory with fermions only in $f_{L}$. Furthermore if $F$ is to be real, then we have to have $D=2+8 n$, while for $F$ pseudoreal $D=6+8 n$.

Starting with an anomaly free theory in higher dimensions, in ref. 20] was given the condition that has to be fulfilled in order to obtain anomaly free theories in four dimensions after dimensional reduction. The condition restricts the allowed embeddings of $R$ into $G$ [21]. For $G=E_{8}$ in ten dimensions it has the form

$$
l(G)=60
$$

where $l(G)$ is the sum over all indices of the $R$ representations appearing in the decomposition of the 248 representation of $E_{8}$ under $E_{8} \supset R \times H$. The normalization is such that the vector representation in eq.(10) which defines the embedding of $R$ into $S O(6)$, has index two.

Next let us obtain the four dimensional effective action. Assuming that the metric is block diagonal, taking into account all the constraints and integrating out the extra coordinates we obtain in four dimensions the following Lagrangian :

$$
A=C \int d^{4} x\left(-\frac{1}{4} F_{\mu \nu}^{t} F^{t \mu \nu}+\frac{1}{2}\left(D_{\mu} \phi_{\alpha}\right)^{t}\left(D^{\mu} \phi^{\alpha}\right)^{t}+V(\phi)+\frac{i}{2} \bar{\psi} \Gamma^{\mu} D_{\mu} \psi-\frac{i}{2} \bar{\psi} \Gamma^{a} D_{a} \psi\right)
$$

where $D_{\mu}=\partial_{\mu}-A_{\mu}$ and $D_{a}=\partial_{a}-\theta_{a}-\phi_{a}$ with $\theta_{a}=\frac{1}{2} \theta_{a b c} \sum^{b c}$ the connection of the coset space, while $C$ is the volume of the coset space. The potential $V(\phi)$ is given by:

$$
V(\phi)=-\frac{1}{4} g^{a c} g^{b d} \operatorname{Tr}\left(f_{a b}^{C} \phi_{C}-\left[\phi_{a}, \phi_{b}\right]\right)\left(f_{c d}^{D} \phi_{D}-\left[\phi_{c}, \phi_{d}\right]\right),
$$

where, $A=1, \ldots, \operatorname{dim} S$ and $f$ ' s are the structure constants appearing in the commutators of the generators of the Lie algebra of $\mathrm{S}$.

The expression (18) for $V(\phi)$ is only formal because $\phi_{a}$ must satisfy the constraints coming from eq.(8),

$$
f_{a i}^{D} \phi_{D}-\left[\phi_{a}, \phi_{i}\right]=0
$$

where the $\phi_{i}$ generate $R_{G}$. These constraints imply that some components $\phi_{a}$ 's are zero, some are constants and the rest can be identified with the genuine Higgs fields. When $V(\phi)$ is expressed in terms of the unconstrained independent Higgs fields, it remains a quartic polynomial which is invariant under gauge transformations of the final gauge group $H$, and its minimum determines the vacuum expectation values of the Higgs fields [22].

In the fermionic Lagrangian the first term is just the kinetic term of the fermions, while the second is the Yukawa term [23]. We note that since $\psi$ is a Majorana-Weyl spinor in ten dimensions the representation in which the fermions are assigned under the gauge group must be real. The second term can be written as

$$
L_{Y}=-\frac{i}{2} \bar{\psi} \Gamma^{a}\left(\partial_{a}-\frac{1}{2} f_{i b c} e_{\gamma}^{i} e_{a}^{\gamma} \Sigma^{b c}-\frac{1}{2} G_{a b c} \Sigma^{b c}-\phi_{a}\right) \psi=\frac{i}{2} \bar{\psi} \Gamma^{a} \nabla_{a} \psi+\bar{\psi} V \psi
$$


where

$$
\begin{aligned}
\nabla_{a} & =-\partial_{a}+\frac{1}{2} f_{i b c} e_{\gamma}^{i} e_{a}^{\gamma} \Sigma^{b c}+\phi_{a} \\
V & =\frac{i}{4} \Gamma^{a} G_{a b c} \Sigma^{b c}
\end{aligned}
$$

and we have used the full connection with torsion [24] given by

$$
\theta_{c b}^{a}=-f_{i b}^{a} e_{\alpha}^{i} e_{c}^{\alpha}-\left(D_{c b}^{a}+\frac{1}{2} T_{c b}^{a}\right)=-f_{i b}^{a} e_{\alpha}^{i} e_{c}^{\alpha}-G_{c b}^{a}
$$

with

$$
D_{c b}^{a}=g^{a d} \frac{1}{2}\left[f_{d b}^{e} g_{e c}+f_{c b}^{e} g_{d e}-f_{c d}^{e} g_{b e}\right] .
$$

A general choice of the torsion tensor which is S-invariant and gives acceptable curvature two-form [13] is

$$
T_{a b c}=2 \tau\left(D_{a b c}+D_{b c a}-D_{c b a}\right),
$$

where $\tau$ is a free parameter. In this case $T_{a b c}$ is fully antisymmetric and when the radii 13 are equal it is proportional to $f_{a b c}$. As we already have noticed, the CSDR constraints tell us that $\partial_{a} \psi=0$. Furthermore we can consider the Lagrangian at the point $y=0$, due to its invariance under $\mathrm{S}$ transformations, and as we showed $e_{\gamma}^{i}=0$ at that point. Therefore eq.(21) becomes just $\nabla_{a}=\phi_{a}$ and the term $\frac{i}{2} \bar{\psi} \Gamma^{a} \nabla_{a} \psi$ in eq.(20) is exactly the Yukawa term.

Let us examine now the last term appearing in eq.(20). One can show easily that the operator $V$ anticommutes with the six-dimensional helicity operator [13]. Furthermore one can show that $V$ commutes with the $T_{i}=-\frac{1}{2} f_{i b c} \Sigma^{b c}\left(T_{i}\right.$ close the $R$-subalgebra of $\left.S O(6)\right)$. In turn we can draw the conclusion, exploiting Schur's lemma, that the non vanishing elements of $V$ are only those which appear in the decomposition of both $S O(6)$ irreps 4 and $\overline{4}$, e.g. the singlets. Since this term is a pure geometric term, we reach the conclusion that the singlets in 4 and $\overline{4}$ will acquire large geometrical masses, a fact that has serious phenomenological implications. In supersymmetric theories defined in higher dimensions, it means that the gauginos obtained in four dimensions after dimensional reduction receive superheavy masses, i.e. supersymmetry is broken at the compactification scale. We note that for symmetric cosets the $V$ operator is absent because $f_{a b}^{c}$ are zero.

\section{Supersymmetry Breaking by Dimensional Reduction over Symmetric Coset Spaces}

Let us first consider the reduction of the supersymmetric $E_{8}$ gauge theory over a symmetric coset space, which is chosen to be the 6-sphere. Therefore $B=S O(7) / S O(6), D=10$ and we choose Weyl-Majorana fermions to belong to the adjoint of $G$. The embedding of $R=S O(6)$ in $E_{8}$ is suggested by the decomposition

$$
\begin{gathered}
E_{8} \supset S O(6) \times S O(10) \\
248=(15,1)+(1,45)+(6,10)+(4,16)+(\overline{4}, \overline{16}) .
\end{gathered}
$$


The $R=S O(6)$ content of the vector and spinor of $S O(7) / S O(6)$ are 6 and 4 respectively. The condition for the anomaly cancelation in four dimensions given in eq.(16) is satisfied and the four-dimensional gauge group is $H=C_{E_{8}}(S O(6))=S O(10)$. The surviving scalars in four dimensions transform as a 10-plet and the surviving fermions as a left handed 16-plet under the gauge group $S O(10)$. Therefore the four dimensional theory is an anomaly free GUT with fermions in a multiplet which is appropriate to describe a family of quark and leptons (including a right handed neutrino), while any sign of the supersymmetry of the original theory has dissappeared in the process of dimensional reduction. Moreover we note that the Higgs content of the theory is not appropriate to make the model phenomenologically attractive. Concerning the spontaneous symmetry breaking of the four dimensional theories resulting from CSDR the following theorems hold :

(i) When $S / R$ is symmetric, the form of the potential of the four-dimensional theory is such that it necessarily leads to a spontaneous breakdown of $H$ [13], and

(ii) When $S$ has an isomorphic image in $G$, the four dimensional gauge group $H$ always breaks down spontaneously to a subgroup $K$ which is the centralizer of $S$ in $G$ [25], [13].

Therefore according to the above theorems in our model the $S O(10)$ gauge symmetry is subject to a spontaneous breakdown, while the final gauge group is $K=C_{E_{8}}(S O(7))=$ $S O(9)$. Thus we see that the scalar field content could be appropriate for the electroweak symmetry breaking but certainly cannot be responsible for the first superstrong breaking at the GUT scale. A mechanism for the superstrong breaking can be provided [26], [13] and then the electroweak breaking due to the Higgs fields is intimately connected to the compactification scale.

\section{Supersymmetry Breaking by Dimensional Reduction over Non Symmetric Coset Spaces}

Now we choose $G=E_{8}$ and $B=G_{2} / S U(3)$, which is a non-symmetric coset space. We use the decomposition

$$
\begin{aligned}
E_{8} & \supset S U(3) \times E_{6} \\
248 & =(8,1)+(1,78)+(3,27)+(\overline{3}, \overline{27}),
\end{aligned}
$$

and we choose $S U(3)$ to be identified with $R$. The $R=S U(3)$ content of $G_{2} / S U(3)$ vector and spinor is $3+\overline{3}$ and $1+3$. The condition (16) for the cancellation of anomalies is satisfied and the resulting four dimensional gauge group is $H=C_{E_{8}}(S U(3))=E_{6}$, which contains fermion and scalar fields transforming as 78, 27 and 27 respectively. Therefore we obtain in four dimensions a supersymmetric anomaly free $E_{6}$ gauge theory with a vector superfield grouping gauge bosons and fermions transforming according to the adjoint and a matter chiral superfield grouping scalars and fermions in the fundamental of the gauge group. Furthermore the very interesting feature of the CSDR over the present coset space that we would like to stress here is that the $\mathcal{N}=1$ supersymmetry of the four dimensional theory is broken by soft terms. More precisely the scalar soft terms appear in the potential of the theory and the gaugino masses come from a geometric (torsion) term as already stated. 
We proceed by calculating these terms. In order to determine the potential we begin by examining the decomposition of the specific $S$ under $R$, i.e.

$$
\begin{aligned}
G_{2} & \supset S U(3) \\
14 & =8+3+\overline{3} .
\end{aligned}
$$

Corresponding to this decomposition we introduce the generators of $G_{2}$

$$
Q_{G_{2}}=\left\{Q^{a}, Q^{\rho}, Q_{\rho}\right\}
$$

where $i=a, \ldots, 8$ correspond to the 8 of $S U(3)$, while $\rho=1,2,3$ correspond to 3 or $\overline{3}$. Then according to the decomposition (28), the non trivial commutation relations of the generators of $G_{2}$ are as follows

$$
\begin{aligned}
& {\left[Q^{a}, Q^{b}\right]=2 i f^{a b c} Q^{c},} \\
& {\left[Q^{a}, Q^{\rho}\right]=-\left(\lambda^{a}\right)_{\sigma}^{\rho} Q^{\sigma},} \\
& {\left[Q^{\rho}, Q_{\sigma}\right]=-\left(\lambda^{a}\right)_{\sigma}^{\rho} Q^{a},} \\
& {\left[Q^{\rho}, Q^{\sigma}\right]=2 \sqrt{\frac{2}{3}} \epsilon^{\rho \sigma \tau} Q_{\tau},}
\end{aligned}
$$

with normalization

$$
\operatorname{Tr} Q^{a} Q^{b}=2 \delta^{a b}, \operatorname{Tr} Q^{\rho} Q_{\sigma}=2 \delta_{\sigma}^{\rho} .
$$

The potential of any theory reduced over $G_{2} / S U(3)$ can be written in terms of the fields

$$
\left\{\phi_{i}, \phi^{\rho}, \phi_{\rho}\right\}
$$

which correspond to the decomposition (28) of $G_{2}$ under $S U(3)$. The $\phi_{i}$ are equal to the generators of the $R$ subgroup. With the help of the commutation relations (30) we find that the potential of any theory reduced over $G_{2} / S U(3)$ is given by $13 \pi$ :

$$
\begin{array}{r}
V(\phi)=8+\frac{4}{3} \operatorname{Tr}\left(\phi^{\rho} \phi_{\rho}\right)-\frac{1}{2}\left(\lambda^{i}\right)_{\sigma}^{\rho} \operatorname{Tr}\left(J_{i}\left[\phi_{\rho}, \phi^{\sigma}\right]\right)+\sqrt{\frac{2}{3}} \epsilon^{\rho \sigma \tau} \operatorname{Tr}\left(\phi_{\tau}\left[\phi_{\rho}, \phi_{\sigma}\right]\right) \\
-\frac{1}{4} \operatorname{Tr}\left(\left[\phi_{\rho}, \phi_{\sigma}\right]\left[\phi^{\rho}, \phi^{\sigma}\right]+\left[\phi^{\rho}, \phi_{\sigma}\right]\left[\phi_{\rho}, \phi^{\sigma}\right]\right) .
\end{array}
$$

Then to proceed with our specific choice $G=E_{8}$ we use the embedding (27) of $R=S U(3)$ in $E_{8}$ and divide accordingly the generators of $E_{8}$

$$
Q_{E_{8}}=\left\{Q^{a}, Q^{\alpha}, Q^{i \rho}, Q_{i \rho}\right\}
$$

with $a=1, \ldots, 8, \alpha=1, \ldots, 78, i=1, \ldots, 27, \rho=1,2,3$. The normalization is

$$
\operatorname{Tr} Q^{a} Q^{b}=2 \delta^{a b}, \operatorname{Tr} Q^{\alpha} Q^{\beta}=12 \delta^{\alpha \beta}, \operatorname{Tr} Q^{i \rho} Q_{j \sigma}=2 \delta_{j}^{i} \delta_{\sigma}^{\rho} .
$$


The non trivial commutation relations of the generators of $E_{8}$ according to the decomposition (27) are the following :

$$
\begin{aligned}
{\left[Q^{a}, Q^{b}\right] } & =2 i f^{a b c} Q^{c}, \\
{\left[Q^{\alpha}, Q^{\beta}\right] } & =2 i g^{\alpha \beta \gamma} Q^{\gamma}, \\
{\left[Q^{a}, Q^{i \rho}\right] } & =-\left(\lambda^{\alpha}\right)_{\sigma}^{\rho} \delta_{j}^{i} Q^{j \sigma}, \\
{\left[Q^{i \rho}, Q^{j \sigma}\right] } & =\frac{1}{\sqrt{6}} \epsilon^{\rho \sigma \tau} d^{i j k} Q_{k \tau}, \\
{\left[Q^{i \rho}, Q_{j \sigma}\right] } & =-\left(\lambda^{a}\right)_{\sigma}^{\rho} \delta_{j}^{i} Q^{a}+\frac{1}{6} \delta_{\sigma}^{\rho}\left(G^{\alpha}\right)_{j}^{i} Q^{\alpha}, \\
{\left[Q^{\alpha}, Q^{i \rho}\right] } & =\left(G^{\alpha}\right)_{j}^{i} \delta_{\sigma}^{\rho} Q^{j \sigma},
\end{aligned}
$$

where $d^{i j k}$, the symmetric invariant $E_{6}$ tensor, and $\left(G^{\alpha}\right)_{j}^{i}$ are defined in ref. 27.

Next we would like to solve the constraints (19) and examine the resulting four dimensional potential in terms of the unconstrained scalar fields $\beta$. The solutions of the constraints in terms of the genuine Higgs fields are

$$
\phi^{a}=Q^{a}, \phi_{\rho}=\beta^{i} Q_{i \rho}, \phi^{\rho}=\beta_{i} Q^{i \rho} .
$$

In turn we can express the Higgs potential in terms of the genuine Higgs field $\beta$ and we find

$$
V(\beta)=8-\frac{40}{3} \beta^{2}-4 d_{i j k} \beta^{i} \beta^{j} \beta^{k}+\beta^{i} \beta^{j} d_{i j k} d^{k l m} \beta_{l} \beta_{m}+\frac{11}{4} \sum_{\alpha} \beta^{i}\left(G^{\alpha}\right)_{i}^{j} \beta_{j} \beta^{k}\left(G^{\alpha}\right)_{k}^{l} \beta_{l} .
$$

From the potential given in eq.(36) we can read directly the F-, D- and scalar soft terms which break softly the supersymmetric theory obtained by CSDR over $G_{2} / S U(3)$. The F-terms are obtained from the superpotential :

$$
\mathcal{W}(B)=\frac{1}{3} d_{i j k} B^{i} B^{j} B^{k}
$$

where $B$ is the chiral superfield whose scalar component is $\beta$. Let us note that the superpotential could also be identified from the relevant Yukawa terms of the fermionic part of the Lagrangian. Correspondingly the D-terms are

$$
D^{\alpha}=\sqrt{\frac{11}{2}} \beta^{i}\left(G^{\alpha}\right)_{i}^{j} \beta_{j} .
$$

The terms in the potential $V(\beta)$ given in eq.(36) that do not result from the $\mathrm{F}$ - and D-terms belong to the soft supersymmetry part of the Lagrangian. These terms are the following

$$
L_{S S B}=-\frac{40}{3} \beta^{2}-4 d_{i j k} \beta^{i} \beta^{j} \beta^{k} .
$$

Finally in order to determine the gaugino mass we calculate the $\mathrm{V}$ operator given in eq.(22). Using eq.(24) we find that

$$
D_{a b c}=\frac{1}{2} f_{a b c}
$$


and in turn the $G_{a b c}=D_{a b c}+\frac{1}{2} T_{a b c}$ is

$$
G_{a b c}=\frac{1}{2}(1-3 \tau) f_{a b c}
$$

In order to obtain the previous results the most general $G_{2}$ invariant metric on $G_{2} / S U(3)$ was used which is $g_{a b}=r \delta_{a b}$.

In addition we need the gamma matrices. In ten dimensions we have $\left[\Gamma^{\mu}, \Gamma^{\nu}\right]=2 \eta^{\mu \nu}$ with $\Gamma^{\mu}=\gamma^{\mu} \otimes I_{8}$ and $\left[\Gamma^{a}, \Gamma^{b}\right]=-2 g^{a b}$, where

$$
\Gamma^{a}=\frac{1}{\sqrt{r}} \gamma_{5} \otimes\left[\begin{array}{cc}
0 & \bar{\gamma}^{a} \\
\bar{\gamma}^{a} & 0
\end{array}\right]
$$

with $a=1,2,3,5,6$ and

$$
\Gamma^{4}=\frac{1}{\sqrt{r}} \gamma_{5} \otimes\left[\begin{array}{cc}
0 & i I_{4} \\
i I_{4} & 0
\end{array}\right] .
$$

The $\bar{\gamma}^{a}$ matrices are given by $\bar{\gamma}^{1}=\sigma^{1} \otimes \sigma^{2}, \bar{\gamma}^{2}=\sigma^{2} \otimes \sigma^{2}, \bar{\gamma}^{3}=-I_{2} \otimes \sigma^{3}, \bar{\gamma}^{5}=\sigma^{3} \otimes \sigma^{2}$, $\bar{\gamma}^{6}=-I_{2} \otimes \sigma^{1}$. Using these matrices we calculate $\Sigma^{a b}=\frac{1}{4}\left[\Gamma^{a}, \Gamma^{b}\right]$. We find that the gauginos acquire a geometrical mass

$$
(1-3 \tau) \frac{6}{\sqrt{3}}
$$

Therefore by reduction of a ten dimensional supersymmetric $E_{8}$ gauge theory over the non symmetric coset space $G_{2} / S U(3)$, we obtain in four dimensions a Lagrangian describing a supersymmetric $E_{6}$ gauge theory as well as its full soft supersymmetry breaking sector. We note that the trilinear and scalar masses soft breaking terms depend only on the radius of the $G_{2} / S U(3)$ while the gaugino has an additional dependence on the possible torsion of the coset space and can be therefore further adjusted.

\section{Conclusion}

In conclusion, we have presented examples of dimensional reduction of a ten-dimensional supersymmetric gauge theory over six-dimensional coset spaces. Our aim was to show, extending and completing previous observations [18], [23], [13], that the CSDR of a supersymmetric gauge theory over non symmetric coset spaces results to a softly broken supersymmetric gauge theory in four dimensions. Thus CSDR provides us with a very interesting mechanism for supersymmetry breaking. A more complete presentation of similar results involving other six-dimensional coset spaces will be given elsewhere due to lack of space here. For completeness we have briefly included in the present examination the CSDR over symmetric coset spaces, which was known that leads to complete breaking of supersymmetry, and can be very useful in attempts to extract physics from p-branes.

We would like to thank P. Forgacs, A. Kehagias, C. Kounnas, G. Koutsoumbas and D. Luest for useful discussions and reading the manuscript. 


\section{References}

[1] S. Dimopoulos and H. Georgi, Phys. Lett. B117, 287(1982); N. Sakai, Z. Phys. C11, $155(1981)$.

[2] J. Wess and B. Zumino, Phys. Lett. B49, 52(1974); J. Iliopoulos and B. Zumino, Nucl. Phys. 76, 310(1974); S. Ferrara, J. Iliopoulos and B. Zumino, Nucl. Phys. 77, 413(1974); K. Fujikawa and W. Lang, Nucl. Phys. 88(1975).

[3] See e.g. I. Antoniadis and A. Sagnotti, Class. Quant. Grav. 17, 939(2000); I. Antoniadis, S. Dimopoulos, A. Pomarol and M. Quiros, Nucl. Phys. B 544, 503(1999); A. Delgado, A. Pomarol and M. Quiros, Phys. Rev. D60, 095008(1999); J. P. Derendinger, L. E. Ibanez and H. P. Nilles, Nucl. Phys. B267, 267(1986); S. Ferrara, C. Kounnas, M. Porrati and F. Zwirner, Nucl. Phys. B 318, 75(1987).

[4] L. Girandello and M. T. Grisaru, Nucl. Phys. B 194, 65(1982); see also H. P. Nilles, Phys. Rept. C 110, 1(1984).

[5] See e.g. I. Antoniadis and K. Benakli, "Large dimensions and string physics in future colliders", hep-ph/0007226, and references therein.

[6] E. Fahri and L. Susskind, Phys. Rept. C 74, 277(1981); C. T. Hill, Proc. of Nagoya Int. Workshop (1996), p. 54, and references therein; ibid., Phys. Lett. B 266, 419(1991); ibid., Nucl. Phys. B 345, 483(1995).

[7] W. Marciano, Phys. Rev. D 21, 315(1980); G. Zoupanos, Phys. Lett. B 129, 315(1983); P. Forgacs and G. Zoupanos, Phys. Lett. B 148, 99(1984); D. Luest et al., Nucl. Phys. B 268, 49(1986).

[8] G. Triantaphyllou, J. Phys. G 26, 99(2000); G. Triantaphyllou and G. Zoupanos, Phys. Lett. B 489, 420(2000).

[9] M. Green and J. H. Schwarz, Phys. Lett. B 149, 117(1984); L. Alvarez-Gaume and E. Witten, Nucl. Phys. B 234, 269(1983).

[10] See e.g. M. B. Green, J. H. Schwarz and E. Witten, Superstring Theory, Cambridge University Press (1987); D. Luest and S. Theisen, Lectures on String Theory, Lecture Notes in Physics, Vol. 346, Springer Verlag, Heidelberg (1989).

[11] P. Horava and E. Witten, Nucl. Phys. B 460, 506(1996); E. Witten, Nucl. Phys. B 471, 135(1996); for a review see C. Munoz in Proc. of Corfu Summer Institute on EPP 1998, hep-th/9906152.

[12] P. Forgacs and N. S. Manton, Commun. Math. Phys. 72, 15(1980); E. Witten, Phys. Rev. Lett. 38, 121(1977).

[13] D. Kapetanakis and G. Zoupanos, Phys. Rept. C 219, 1(1992). 
[14] Y. Kubyshin, J. M. Mourao, G. Rudolph and I. P. Volobujev, Lecture notes in Physics, Vol. 349, Springer Verlag, Heidelberg (1989).

[15] T. R. Taylor and G. Veneziano, Phys. Lett. B 212, 147(1988).

[16] K. R. Dienes, E. Dudas and T. Gherghetta, Nucl. Phys. B 537, 47(1999).

[17] T. Kobayashi, J. Kubo, M. Mondragon and G. Zoupanos, Nucl. Phys. B 550, 99(1999);

J. Kubo, H. Terao and G. Zoupanos, Nucl. Phys. B 574, 495(2000); ibid., hepph/0010069.

[18] G. Chapline and R. Slansky, Nucl. Phys. B 204, 461(1982).

[19] N. S. Manton, Nucl. Phys. B193, 502(1981); C. Wetterich, Nucl. Phys. B222, 20 (1985); L. Palla, Z. Phys. C 24, 345(1983); K. Pilch and A. N. Schellekens, J. Math. Phys. 25, 3455(1984); P. Forgacs, Z. Horvath and L. Palla, Z. Phys. C 30, 261(1986); K. J. Barnes, P. Forgacs, M. Surridge and G. Zoupanos, Z. Phys. C 33, 427(1987).

[20] E. Witten, Phys. Lett. B144, 351(1984).

[21] K. Pilch and A. N. Schellekens, Nucl. Phys. B259, 673(1985); D. Luest, Nucl. Phys. B276, 220(1985); D. Kapetanakis and G. Zoupanos, Phys. Lett. B249, 66(1990).

[22] G. Chapline and N. S. Manton, Nucl. Phys. B184, 391(1981); K. Farakos, G. Koutsoumbas, M. Surridge and G. Zoupanos, Nucl. Phys. B291, 128(1987); ibid., Phys. Lett. B191, 135(1987); Y. A. Kubyshin, J. M. Mourao, I. P. Volobujev, Int. J. Mod. Phys. A4, 151(1989); F. A. Bais, K. J. Barnes, P. Forgacs and G. Zoupanos, Nucl. Phys. B 263, 557(1986).

[23] D. Kapetanakis and G. Zoupanos, Phys. Lett. B249, 73(1990); ibid., Z. Phys. C56, 91(1992).

[24] F. Muller-Hoissen and R. Stuckl, Class. Quant. Grav. 5, 27(1988); N. A. Batakis, et al. Phys. Lett. B 220, 513(1989).

[25] J. Harnad, S. Shnider and L. Vinet, J. Math. Phys. 20, 931(1979); 21, 2719(1980);

J. Harnad, S. Shnider and J. Taffel, Lett. Math. Phys. 4, 107(1980).

[26] Y. Hosotani, Phys. Lett. B126, 193(1983); G. Zoupanos, Phys. Lett. B 201, 301(1988);

N. G. Kozimirov, V. A. Kuzmin and I. I. Tkachev, Sov. J. Nucl. Phys. 49, 164(1989);

D. Kapetanakis and G. Zoupanos, Phys. Lett. B 232, 104(1989).

[27] T. W. Kephart and M. T. Vaughn, Annals of Physics 145, 162(1983). 